\title{
A NOTE ON SINGULARITIES IN SEMILINEAR PROBLEMS
}

\author{
MOHAMMED GUEDDA AND MOKHTAR KIRANE
}

\begin{abstract}
We consider the equation $\Delta u-\frac{1}{2} x . \Delta u-\frac{u}{q-1}+u^{q}=0$, for $q>1$. We study the isolated singularities and present a nonlinear technique, and give a complete classification.
\end{abstract}

\section{INTRODUCTION}

In this note we study the isolated singularities of the positive solutions of the following nonlinear equation:

$$
\Delta u-\frac{1}{2} x \cdot \nabla u-\frac{u}{q-1}+u^{q}=0 .
$$

Let $\Omega$ be an open subset of $\mathbf{R}^{N}, N \geq 2$, containing $0, \Omega^{\prime}=\Omega \backslash\{0\}$ and $q>1$. We are concerned with the following question:

If $u \in C^{2}\left(\Omega^{\prime}\right)$ is a positive solution of (1.1) in $\Omega^{\prime}$, what can be said about $u(x)$ and about the equation as $|x| \rightarrow 0$ ?

It is well known [15] that (1.1) has no nontrivial globally bounded solution for $N=1,2$ or $N>2, q \leq \frac{N+2}{N-2}$ except the constant solution $u=\left(\frac{1}{q-1}\right)^{1 /(q-1)}$.

If we look for a specific solutions of (1.1) under the form

$$
u(r)=\alpha r^{\beta},
$$

then we get

$$
\beta=-\frac{1}{q-1} \text { and } \alpha=\lambda_{N, q}=\left\{\frac{2}{q-1}\left(N-\frac{2 q}{q-1}\right)\right\}^{1 /(q-1)}
$$

where it is clear that $\lambda_{N, q}$ only exists when $N>2$ and $q>\frac{N}{N-2}$. It follows by substitution that $u(r)=\lambda_{N, q} r^{\beta}$ is also a solution of the Emden-Fowler equation

$$
\Delta w+w^{q}=0 .
$$

This equation has been intensively studied. When $N>2$ two critical values $\frac{N}{N-2}$ and $\frac{N+2}{N-2}$ appear. The first studies in the radial case are due to Emden, then Fowler [3, 5, 6], Brezis and Lions [2] and Lions [22] (see also [18, 1]).

Let us briefly describe our results. We have two cases.

Received by the editors July 6, 1994 and, in revised form, October 21, 1994.

1991 Mathematics Subject Classification. Primary 35J65.

The first author was supported by LAMIFA F Amiens. 
First case. $1<q<\frac{N}{N-2}$. Then any positive solution of (1.1) in $\Omega^{\prime}$ satisfies the equation $-\Delta u+\frac{1}{2} x . \nabla u+\frac{u}{q-1}-u^{q}=\alpha \delta_{0}$ in $D^{\prime}(\Omega)$ with $\alpha \geq 0$. Furthermore if $\alpha=0$, then the "singularity is removable".

Second case. $\frac{N}{N-2}<q<\frac{N+2}{N-2}$. Then for any positive radial solution of (1.1) in $\Omega^{\prime}$ we have $\operatorname{Lim} r^{2 /(q-1)} u(r)=l \in\left\{0, \lambda_{N, q}\right\}$ as $r \rightarrow 0$, where $r=|x|$. If $l=0$ and $q>2$, the "singularity is removable". Note that if $q \geq \frac{N}{N-2}$, any solution of (1.1) satisfies

$$
-\Delta u+\frac{1}{2} x \cdot \nabla u+\frac{u}{q-1}-u^{q}=0, \quad \text { in } D^{\prime}(\Omega) .
$$

\section{MAIN RESUltS}

Let $N>2$ and set $\Omega=B_{R}(0)=\left\{x \in \mathbf{R}^{N},|x|<R\right\}, R>0$ and $\Omega^{\prime}=$ $\Omega \backslash\{0\}$. Our main result is the following:

Theorem 2.1. Assume that $u \in L_{\text {Loc }}^{1}\left(\Omega^{\prime}\right)$,

$$
\begin{gathered}
\Delta u-\frac{1}{2} x \cdot \nabla u \in L_{\text {Loc }}^{1}\left(\Omega^{\prime}\right), \quad \text { in } D^{\prime}\left(\Omega^{\prime}\right), \\
u \geq 0, \quad \Delta u-\frac{1}{2} x . \nabla u \leq a u+f \text { a.e. in } \Omega,
\end{gathered}
$$

where $a$ is a nonnegative constant and $f \in L_{\text {Loc }}^{1}(\Omega)$. Then

$$
u \in L_{\mathrm{Loc}}^{1}(\Omega) \text {, }
$$

and there exists $h \in L_{\mathrm{Loc}}^{1}(\Omega)$, and $\alpha \geq 0$ such that

$$
-\Delta u+\frac{1}{2} x \cdot \nabla u=h+\alpha \delta_{0} \quad \text { in } D^{\prime}(\Omega) \text {. }
$$

For the proof, we use a nonlinear technique introduced by Serrin [23], [24] and a linear method by H. Brezis and P. L. Lions [2], [22].

The proof is divided into five steps.

Step 1. We claim that

where $x=(r, \sigma), r=|x|$.

$$
x . \nabla u=r \frac{\partial u}{\partial r}
$$

Proof of the claim. Writing

$$
\begin{aligned}
& x . \nabla u=\sum_{i=1}^{N} x_{i}\left(\frac{\partial u}{\partial r} \frac{\partial r}{\partial x_{i}}+\sum_{j=1}^{N-1} \frac{\partial u}{\partial \sigma_{j}} \frac{\partial \sigma_{j}}{\partial x_{i}}\right) \\
& x . \nabla u=r \frac{\partial u(r, \sigma)}{\partial r}+\sum_{j=1}^{N-1} \frac{\partial u}{\partial \sigma_{j}} \sum_{i=1}^{N} x_{i} \frac{\partial \sigma_{j}}{\partial x_{i}}
\end{aligned}
$$

as in [17], we have

$$
\frac{\partial \sigma_{j}}{\partial x_{i}}= \begin{cases}\frac{x_{i} x_{j+1}}{r_{j} r_{j+1}^{2}} & \text { if } i \leq j \\ -\frac{r_{j}}{r_{j+1}^{2}} & \text { if } i=j+1 \\ 0 & \text { if } i>j+1\end{cases}
$$


where $r_{j}^{2}=x_{1}^{2}+x_{2}^{2}+\cdots+x_{j}^{2}, r_{N}^{2}=r^{2}$. Thus

$$
\sum_{i=1}^{N} x_{i} \frac{\partial \sigma_{j}}{\partial x_{i}}=0
$$

And then

$$
x . \nabla u=r \frac{\partial u}{\partial r} .
$$

Step 2. $u \in L_{\text {loc }}^{1}(\Omega)$. We begin by considering the average

$$
\bar{u}(r)=\frac{1}{\omega_{N-1}} \int_{S^{N-1}} u(r, \sigma) d \sigma, \quad 0<r<R,
$$

where $\omega_{N-1}$ is the volume of the sphere $S^{N-1}$. It follows from (2.1), (2.2) and (2.5) that

$$
\begin{gathered}
\Delta \bar{u}-\frac{1}{2} r \bar{u}_{r} \in L_{\mathrm{Loc}}^{1}(0, R), \quad \bar{u} \geq 0, \\
\Delta \bar{u}-\frac{1}{2} r \bar{u}_{r} \leq a \bar{u}+\bar{f} \quad \text { on }(0, R),
\end{gathered}
$$

and

$$
\frac{1}{r^{N-1}}\left(r^{N-1} K(r) \bar{u}_{r}\right)_{r} \leq a \bar{u}+\bar{f}, \quad \text { for } r \in(0, R),
$$

where $K(r)=e^{-r^{2} / 4}$. In particular $\bar{u} \in C^{1}(0, R)$.

Let $R^{\prime}<R$ be fixed. Integrating (2.8) over $\left(r, R^{\prime}\right)$ we find as in [2] that

$$
u \in L_{\text {loc }}^{1}(\Omega)
$$

and

$$
\bar{u}(r) \leq \frac{C}{r^{N-2}}+C .
$$

Step 3. Let $g(x)=-\operatorname{div}(K(x) \nabla u)$ a.e. in $\Omega$, where $K(x)=e^{-|x|^{2} / 4}$. Then $g \in L_{\text {loc }}^{1}(\Omega)$ and for any $\varphi \in D(\Omega), 0 \leq \varphi \leq 1, \varphi \equiv 1$ near $x=0$,

$$
\int_{\Omega} g \varphi^{2} d x \leq-\int_{\Omega} u \cdot \operatorname{div}\left(K(x) \nabla \varphi^{2}\right) d x .
$$

Proof of the step 3. From the definition of $g$ we have

$$
\int_{\Omega} g \psi d x=\int_{\Omega} K(x) \nabla u \nabla \psi d x,
$$

for any $\psi \in W^{1, \infty}\left(\Omega^{\prime}\right)$ with compact support in $\Omega^{\prime}$. Set

$$
P_{k}(t)= \begin{cases}1 & \text { if } t>1+k, \\ t-k & \text { if } k \leq t \leq 1+k, \\ 0 & \text { if } t<k\end{cases}
$$

for any $t \geq 0$ and $k \geq 0$. 
Let $0<\rho<R$ and $\varepsilon<\frac{\rho}{2}$. Let $\varphi \in D(\Omega)$ be such that $0 \leq \varphi \leq 1, \varphi \equiv 1$ on $B_{\rho}=\left\{x \in \mathbf{R}^{N},|x|<\rho\right\}$ and $\psi_{\varepsilon}=\eta_{\varepsilon} \varphi$ with $\eta_{\varepsilon} \in C^{\infty}(\Omega), 0 \leq \eta_{\varepsilon} \leq 1, \eta_{\varepsilon} \equiv$ 0 if $|x|<\varepsilon, \eta_{\varepsilon}(x) \equiv 1$ if $|x|>2 \varepsilon$ and $\left|\nabla \eta_{\varepsilon}\right| \leq \frac{c}{\varepsilon}$. We have, from (2.12),

$$
\begin{gathered}
\int_{\Omega} g\left(1-P_{k}(u)\right) \psi_{\varepsilon}^{2} d x+\int_{\{k<u<1+k\}} K(x)|\nabla u|^{2} \psi_{\varepsilon}^{2} d x \\
=\int_{\Omega}\left(1-P_{k}(u)\right) K \nabla u \nabla \psi_{\varepsilon}^{2} d x .
\end{gathered}
$$

Since

$$
\sum_{k=0}^{n}\left(1-P_{k}(t)\right)^{+}=(n+1-t)^{+}
$$

we have

$$
\begin{aligned}
& \int_{\{u<n+1\}} g(n+1-u)^{+} \psi_{\varepsilon}^{2} d x+\int_{\{u<1+n\}} K(x)|\nabla u|^{2} \psi_{\varepsilon}^{2} d x \\
& \quad \leq \int_{\{u<n+1\}}(n+1-u) K \nabla u \nabla \varphi^{2} \eta_{\varepsilon}^{2} d x \\
& \quad+2 \int_{\{u<1+n\} \cap B_{2 \varepsilon}}(n+1-u) K(x)|\nabla u| \psi_{\varepsilon}\left|\nabla \eta_{\varepsilon}\right| d x .
\end{aligned}
$$

Now for any real $h>0$, we have $n+1-u(x)>n+1 \frac{h}{h+1}$ a.e. in $\left\{u<\frac{n+1}{h+1}\right\}$. Thus, dividing by $n+1$ and using the Hölder inequality, for any $\beta>0$,

$$
\begin{aligned}
\frac{h}{h+1} & \int_{\left\{u<\frac{n+1}{h+1}\right\}} g \psi_{\varepsilon}^{2}+\frac{1}{n+1} \int_{\{u<1+n\}} K|\nabla u|^{2} \psi_{\varepsilon}^{2} \\
\leq & \int_{\{u<1+n\}}\left(1-\frac{u}{n+1}\right) K \nabla u \nabla\left(\varphi^{2}\right) \eta_{\varepsilon}^{2} \\
& +\beta^{2} \int_{\{u<1+n\}} K(x)|\nabla u|^{2} \psi_{\varepsilon}^{2}+\beta^{-2} \int_{B_{2 \varepsilon}} K(x)\left|\nabla \eta_{\varepsilon}\right|^{2} .
\end{aligned}
$$

Let $\beta^{2}=\frac{1}{2(n+1)}$; then

$$
\begin{aligned}
& \frac{h}{h+1} \int_{\left\{u<\frac{n+1}{h+1}\right\}} g \psi_{\varepsilon}^{2}+\frac{1}{2(n+1)} \int_{\{u<1+n\}} K|\nabla u|^{2} \psi_{\varepsilon}^{2} \\
& \quad \leq \int_{\{u<1+n\}}\left(1-\frac{u}{n+1}\right) K \nabla u \nabla\left(\varphi^{2}\right) \eta_{\varepsilon}^{2}+2(n+1) C \varepsilon^{N-2} .
\end{aligned}
$$

From Fatou's Lemma-which is valid since $g \geq-a u-f \in L_{\text {loc }}^{2}(\Omega)$-we deduce as $\varepsilon \rightarrow 0, n \rightarrow+\infty, h \rightarrow+\infty$ that $g \varphi^{2} \in L^{1}(\Omega)$ and satisfies $(2.11)$.

Step 4. Now since $u \in L^{1}(\Omega)$, we can define the distribution

$$
T=-\operatorname{div}(K \nabla u)-g \text { in } D^{\prime}(\Omega) .
$$

Then as in [2], we have

$$
T=\sum_{|p| \leq m} c_{p} D^{p} \delta_{0}
$$

Let $\psi \in D\left(B_{R}\right)$ be any fixed function such that

$$
(-1)^{|p|} D^{p} \psi(0)=c_{p} \quad \text { for every }|p| \leq m
$$


and

$$
\psi_{\varepsilon}(x)=\psi\left(\frac{x}{\varepsilon}\right)
$$

Then

$$
\int_{B_{R}} u \operatorname{div}\left(K \nabla \psi_{\varepsilon}\right)=\int_{B_{R}} g \psi_{\varepsilon}+\sum_{|p| \leq m} \frac{c_{p}^{2}}{\varepsilon^{|p|}} .
$$

On the other hand we have

$$
\left|\int_{B_{R}} u \operatorname{div}\left(K \nabla \psi_{\varepsilon}\right)\right| \leq \frac{C}{\varepsilon^{2}} \int_{B_{R \varepsilon}} u d x+C R \int_{B_{R \varepsilon}} u d x
$$

and therefore

$$
\left|\int_{B_{R}} u \operatorname{div}\left(K \nabla \psi_{\varepsilon}\right)\right| \leq \frac{C}{\varepsilon^{2}} \int_{0}^{R \varepsilon} \bar{u} r^{n-1} d r+C R \int_{0}^{R \varepsilon} \bar{u} r^{n-1} d r .
$$

We deduce from (2.10) that $\left|\int_{B_{R}} u \operatorname{div}\left(K \nabla \psi_{\varepsilon}\right)\right| \leq C$. Comparing this with (2.14) we conclude that $c_{p}=0$ when $|p| \geq 1$.

Finally we choose $\eta \in D(\Omega), 0 \leq \eta \leq 1$, and $\eta \equiv 1$ near $x=0$.

We have

$$
\left\langle T, \eta^{2}\right\rangle=c_{0}=\int_{\Omega} u \operatorname{div}\left(K \nabla \eta^{2}\right)-\int_{\Omega} g \eta^{2},
$$

and hence $c_{0} \geq 0$ from (2.11).

Step 5. The end of the proof of Theorem 2.1 .

Let $h(x)=g(x) e^{|x| / 4}$ a.e. in $\Omega$; then $h \in L_{\text {loc }}^{1}(\Omega)$ and $-\Delta u+\frac{1}{2} x . \nabla u=$ $h+c_{0} \delta_{0}$ in $D^{\prime}(\Omega)$.

\section{THE SUbCRITICAL CASE}

Now we return to equation (1.1). Let $\mu$ be the fundamental harmonic function in $\mathbf{R}^{N} \backslash\{0\}, N>2$, that is

$$
\mu(x)=\frac{1}{N(N-2) \omega_{N}}|x|^{2-N} .
$$

Theorem 3.1. Let $N>2,1<q<\frac{N}{N-2}$. Let $u \in C^{2}\left(\Omega^{\prime}\right)$ be a nonnegative solution of equation

$$
\Delta u-\frac{1}{2} x \cdot \nabla u-\frac{u}{q-1}+u^{q}=0 \quad \text { in } \Omega^{\prime}=\Omega \backslash\{0\} .
$$

Then

(i) either $u$ can be extended to a smooth solution of (3.2) in $\Omega$, or

(ii) there exists $\alpha>0$ such that $\lim _{x \rightarrow 0} \frac{u(x)}{\mu(x)}=\alpha$ and $u$ satisfies the equation

$$
-\Delta u+\frac{1}{2} x \cdot \nabla u+\frac{u}{q-1}-u^{q}=\alpha \delta_{0} \quad \text { in } D^{\prime}(\Omega) .
$$

Proof. From (3.2) we have $\Delta u-\frac{1}{2} x . \nabla u \leq \frac{u}{q-1}$; hence from Theorem 2.1 we have

$$
-\Delta u+\frac{1}{2} x \cdot \nabla u+\frac{u}{q-1}-u^{q}=\beta \delta_{0}, \quad u \in L_{\mathrm{loc}}^{1}(\Omega), u^{q} \in L_{\mathrm{loc}}^{1}(\Omega) .
$$


Moreover equation (3.2) can be written in the form

$$
\operatorname{div}(K \nabla u)+d(x) u=0 \text { in } \Omega^{\prime}
$$

where $d(x)=k(x)\left(u^{q-1}-\frac{1}{q-1}\right)$. Since $q<\frac{N}{N-2}$ we can find an $\varepsilon>0$ such that $d \in L_{\text {loc }}^{N /(2-\varepsilon)}(\Omega)$. We then deduce from [24] that if $u$ is singular at 0 there exists a constant $C>0$ such that

$$
C \mu \leq u \leq \frac{1}{C} \mu \text { near } 0 .
$$

As in Guedda-Veron [18], we prove by scaling that there is an $\alpha>0$ such that

$$
\lim _{x \rightarrow 0} \frac{u(x)}{\mu(x)}=\alpha
$$

we get that

$$
\lim _{x \rightarrow 0}|x|^{N-1} \nabla u(x)=\frac{\alpha}{N \omega_{N}} \xi, \quad \text { where } \xi=\lim _{x \rightarrow 0} \frac{x}{|x|}
$$

and then (3.3).

\section{THE SUPER CRITICAL CASE}

We still assume that $\Omega=B_{R}(0)=\left\{x \in \mathbf{R}^{N},|x|<R\right\}$ and $\Omega^{\prime}=\Omega \backslash\{0\}$. In this section we present some results concerning the isolated singularities of the positive solutions of (1.1), that is, of

$$
-\Delta u+\frac{1}{2} x \cdot \nabla u+\frac{u}{q-1}-u^{q}=0 \text { in } \Omega^{\prime}
$$

where

$$
\frac{N}{N-2}<q<\frac{N+2}{N-2} \text { and } N>2 .
$$

If we look for the solution of (3.2) under the form $u(x)=u(|x|)=\alpha|x|^{\beta}$, then we get

$$
\beta=-\frac{1}{q-1} \quad \text { and } \quad \alpha=\lambda_{N, q}=\left(\frac{2}{q-1}\left(N-\frac{2 q}{q-1}\right)\right)^{1 /(q-1)} .
$$

Theorem 4.1. Let $N>2, q \geq \frac{N}{N-2}$. Let $u \in C^{2}\left(\Omega^{\prime}\right)$ be a nonnegative solution of (4.1) in $D^{\prime}\left(\Omega^{\prime}\right)$. Then we have $\alpha=0$ in (3.3), i.e.

$$
-\Delta u+\frac{1}{2} x . \nabla u+\frac{u}{q-1}-u^{q}=0 \text { in } D^{\prime}(\Omega) .
$$

Theorem 4.2. Under the assumptions of Theorem 4.1, if

$$
u^{(q-1) N / 2} \in L_{\mathrm{loc}}^{1}(\Omega),
$$

then $u$ can be extended to $\Omega$ as a solution of (4.1) in $\Omega$.

The proofs of Theorems 4.1 and 4.2 are the same as in Guedda and Veron [18] and they are omitted.

The main result of this section is the following 
Theorem 4.3. Let $N>2$ and $\frac{N}{N-2}<q<\frac{N+2}{N-2}$ and $u$ be a positive radial solution of (4.1).

Then we have

(i) $r^{2 /(q-1)} u(r)$ has a limit $l$ as $r \rightarrow 0$ and $l \in\left\{0, \lambda_{N, q}\right\}$.

(ii) If $l=0$ and $q>2$, then $u$ can be extended to $\Omega$ as a $C^{2}(\Omega)$ solution of $(4.1)$ in $\Omega$.

We need the following

Lemma 4.1. Let $u$ be a positive radial solution of (4.1) in $\Omega^{\prime}$. Then $r^{2 /(q-1)} u(r)$ is bounded as $r \rightarrow 0$.

Proof. Let

$$
t=\log \left(r^{\gamma}\right), \quad v(t)=r^{2 /(q-1)} u(r), \quad \gamma=\frac{4}{q-1}+2-N>0 ;
$$

then (4.1) takes the equivalent form

$$
\gamma^{2}\left(v_{t t}+v_{t}\right)+\frac{1}{2} \gamma e^{-2 t / \gamma} v_{t}+\lambda v+v^{q}=0
$$

where $\lambda=\frac{2}{q-1}\left(\frac{2}{q-1}+2-N\right)<0$. Let

$$
L(t)=\frac{1}{2} \gamma^{2} v_{t}^{2}+\lambda \frac{v^{2}}{2}+\frac{v^{q+1}}{q+1}, \quad \text { for } t \in[0,+\infty[.
$$

From (4.6) the function $L$ is nonincreasing. Hence for any $t_{0} \leq t<+\infty$

$$
\frac{1}{2} \gamma^{2} v_{t}^{2}+\lambda \frac{v^{2}}{2}+\frac{v^{q+1}}{q+1} \leq L\left(t_{0}\right) \text {. }
$$

Hence $v$ is bounded, as $q>1$.

Lemma 4.2. Suppose $\frac{N}{N-2}<q<\frac{N+2}{N-2}$. Let $v$ be any solution of (4.6). Then $\lim _{t \rightarrow+\infty} v_{t}=0$ and $v$ has a limit $l$ at $+\infty$ and

$$
l\left(\lambda+l^{q-1}\right)=0 .
$$

Proof. Using (4.6), (4.7) and Lemma 4.1 we have

$$
v, v_{t}, v_{t t} \text { and } v_{t t t} \text { are bounded. }
$$

Hence $v_{t} \in L^{2}\left(t_{0},+\infty\right)$. This implies $v_{t t} \in L^{2}\left(t_{0},+\infty\right)$.

It follows from this and (4.9) that

$$
\lim _{t \rightarrow+\infty} v_{t}=\lim _{t \rightarrow+\infty} v_{t t}=0
$$

For any $t \geq \tau \geq t_{0}$, we have

$$
|v(t)-v(\tau)| \leq\left\|v_{t}\right\|_{L^{2}\left(t_{0},+\infty\right)} \cdot \sqrt{(t-\tau)},
$$

and hence $v$ has a limit $l$ at $+\infty$. From (4.6) we get (4.8).

Proof of Theorem 4.3. From Lemmas 4.1 and $4.2 r^{2 /(q-1)} u(r)$ has a limit $l$ where $l=0$ or $\lambda_{N, q}$.

Let $v$ be as in (4.5). Assume that $l=0$. The proof of (ii) is divided into two steps. 
Step 1. Assume $v$ decreases to 0 as $t$ tends to $+\infty$; then

$$
v(t) \leq C e^{-t},
$$

for $t \geq 0$ and some $C>0$. In fact, from (4.6) we have

$$
\gamma^{2}\left(v_{t}+v\right)=\int_{t}^{+\infty} \frac{1}{2} \gamma e^{-2 t / \gamma} v_{s}+\lambda v+v^{q} d s .
$$

Since $v$ and $v_{t}$ are bounded, $\omega(t)=\frac{1}{2} \gamma e^{-2 t / \gamma} v_{s}+\lambda v+v^{q}$ is integrable; then $\lambda v+v^{q}$ is integrable and then $v$ is integrable. Since $v_{t} \leq 0$ and $\lambda<0$, we have

$$
\gamma^{2}\left(v_{t}+v\right) \leq \int_{t}^{+\infty} v^{q} d s \leq v^{q-1} \int_{t}^{+\infty} v d s
$$

then

$$
v_{t}+v \leq C v^{q-1}, \quad \text { for } t \text { large }
$$

which implies, if $q>2,(4.10)$. Moreover, Theorem 4.2 implies that if $v$ satisfies (4.10), then $u$ can be extended to $\Omega$ as a regular solution of (1.1) in $\Omega$.

Step 2. Assume that $v$ is not asymptotically monotone. Then there exists a sequence $\left\{t_{n}\right\}$ such that $v_{t}\left(t_{n}\right)=0, v\left(t_{2 n}\right)$ is local minimum and $v\left(t_{2 n+1}\right)$ is local maximum, and (from the equation)

$$
0<v\left(t_{2 n}\right)<\lambda_{N, q}<v\left(t_{2 n+1}\right)
$$

which is not possible since $\lim _{t \rightarrow+\infty} v(t)=0$, which ends the proof.

\section{REFERENCES}

1. M. F. Bidaut-Veron, Local and global behaviour of solutions of quasilinear equations of Emden-Fowler equations type, Arch. Rational Mech. Anal. 107 (1989), 293-324.

2. H. Brezis and P. L. Lions, A note on isolated singularities for linear elliptic equations, J. Math. Anal. Appl. 7A (1981), 263-266.

3. R. Emden, Gaskugeln, Anwendungen der mechanischen Warmentheorie auf Kosmologie und meteorologische Probleme, Chap. XII, Teubner, Leipzig, 1907.

4. M. Escobedo and O. Kavian, Variational problems related to self-similar solutions of the heat equation, Nonlinear Anal., Theory, Methods Appl. 11 (1987), 1103-1133.

5. R. H. Fowler, The form near infinity of real continuous solutions of a certain differential equation of second order, Quart. J. Math. 45 (1914), 289-350.

6. _ The solutions of Emden's and similar differential equations, Monthly Notices Roy. Astronom. Soc. 91 (1920), 63-91.

7. _ Further studies on Emden's and similar differential equations, Quart J. Math. 2 (1931), 259-288.

8. A. Friedman, Remarks on nonlinear parabolic equations, Applications of Nonlinear Partial Differential Equations in Mathematical Physics, Amer. Math. Soc., Providence, RI, 1965, pp. 3-23.

9. A. Friedman and J. B. McLeod, Blow-up of positive solutions of semilinear heat equations, Indiana Univ. Math. J. 34 (1985), 425-447.

10. A. Friedman, Blow-up of solutions of non linear parabolic equations, Nonlinear Diffusion Equations and their Equilibrium States, vol. 1 (W.-H. Ni et al., eds), Springer-Verlag, 1988, pp. 301-318.

11. H. Fujita, On the blowing up of solutions to the Cauchy problem for $u_{t}=\Delta u+u^{1+\alpha}$, J. Fac. Sci. Univ. Tokyo Sect. IA 13 (1966), 109-124. 
12. V. A. Galaktionov, S. P. Kurdymov and A. A. Samarskii, Asymptotic stability of invariant solutions of nonlinear heat-condiction equations with sources, translation of Differentsial'nye Uravneniya 20 (1981), 461-476.

13. V. A. Galaktionov, Proof of the localization of unbounded solutions of the nonlinear parabolic equation, Differentsial'nye Uravneniya 21 (1985), 15-23.

14. B. Gidas and J. Spruck, Global and local behaviour of positive solutions of nonlinear elliptic equations, Comm. Pure Appl. Math. 34 (1981), 525-598.

15. Y. Giga and R. V. Kohn, Asymptotically self-similar blow-up for semilinear heat equations, Comm. Pure Appl. Math. 38 (1985), 297-319.

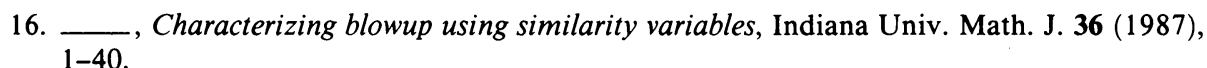

17. A. Gmira, Comportements asymptotiques et singularités des solutions de problèmes quasilinéaires, Doctorat d'Etat, Université de Tours, 1989.

18. M. Guedda and L. Veron, Local and global properties of solutions of quasilinear equations, J. Differential Equations 76 (1988), 159-189.

19. M. Guedda, Propriétés locales et globales des solutions d'équations quasilinéaires elliptiques, Ph.D. thesis, Université de Tours, 1987.

20. R. Hadiji and M. Guedda, Some remarks on Sobolev inequality (to appear).

21. S. Kaplan, On the growth of solutions of quasilinear parabolic equations, Comm. Pure Appl. Math. 16 (1963), 305-330.

22. P. L. Lions, Isolated singularities in semilinear problems, J. Differential Equations 38 (1980), 441-450.

23. J. Serrin, Local behaviour of solutions of quasilinear equations, Acta Math. 111 (1964), 247-302.

24. __ Isolated singularities of solutions of quasilinear equations, Acta Math. 113 (1965), 219-240.

25. L. Veron, Singularities of some quasilinear equations, Nonlinear Diffusion Equations and their Equilibrium States (W. M. Ni, L. A. Peletier, and J. Serrin, eds.), M.S.R.I. Publ., Springer-Verlag, New York and Berlin, 1988.

Université de Picardie Jules Verne, Faculté de Mathematiques et d'Informatique, 33, Rue Saint Leu, 80039 amiens Cedex 01 France

E-mail address: guedda@crihan.fr

Université de Annaba, Institut de Mathématiques, B.P. 12 Annaba, 2300 Algérie 